\title{
Local Mode Excitation for Time Resolved Unimolecular Decay Studies $\dagger$
}

\author{
T. R. RIZZO, B. D. CANNON, E. S. McGINLEY AND F. F. CRIM $\ddagger$ \\ Department of Chemistry, University of Wisconsin, Madison, Wisconsin 53706
}

Laser excitation of overtone vibrations is a useful technique for selectively preparing molecules for state-selected unimolecular reaction studies. In addition, these studies provide new data on overtone vibration transitions in the excited molecules. Data obtained from state-selected unimolecular decay experiments and photoacoustic measurements provide local mode parameters for overtone vibrations in tetramethyldioxetane, $t$-butylalcohol, $t$-butylhydroperoxide, and hydrogen peroxide.

\section{INTRODUCTION}

Time and state resolved studies of unimolecular reactions hold the possibility of uncovering selective chemistry and revealing details of intramolecular energy redistribution but require a highly specific excitation to prepare the reactant molecules in a well characterized initial state. Laser excitation of overtone vibrations is appropriately specific and, in carefully chosen systems, is suitable for use in direct measurements of unimolecular decay rates and product energy partitioning. We have combined this excitation technique with timeresolved spectroscopic detection to measure directly the unimolecular reaction rate in tetramethyldioxetane ${ }^{1}$ and $t$-butylhydroperoxide ${ }^{2}$ and to explore the energy partitioning in the latter molecule. We have also determined the product energy partitioning in hydrogen peroxide and in its partially deuterated analogue. ${ }^{3}$

All of these overtone vibration initiated unimolecular reaction studies require a knowledge of the appropriate transitions in the

† Supported by Office of Basic Energy Sciences of the Department of Energy.

$\ddagger$ Alfred P. Sloan Research Fellow, and Camille and Henry Dreyfus Teacher-Scholar. 
molecule of interest. The high overtone vibrations are well described as local modes of molecular vibrations in which a light atom stretching motion in a polyatomic molecule behaves much like an isolated diatomic oscillator. ${ }^{4,5}$ The Birge-Sponer relation for diatomic molecules, $\tilde{\nu}=A v+B v^{2}$, fits the transition frequencies of the local mode vibrations where $\tilde{\nu}$ is the transition energy, $v$ is the vibrational quantum number, $B$ is the anharmonicity, and $A-B$ is the mechanical frequency of the oscillator. ${ }^{6}$ The parameters for a particular motion such as a $\mathrm{CH}$ or $\mathrm{OH}$ stretch are roughly transferable among molecules, but their exact values reflect the immediate environment of the oscillator.

Our overtone vibration initiated unimolecular decay studies provide data on local mode transitions in addition to requiring knowledge of these transitions. Monitoring the unimolecular reaction products as a function of overtone vibration excitation wavelength reveals the local mode spectrum for those molecules which react. This paper describes the local mode excitation spectra of a cyclic peroxide, tetramethyldioxetane, and two hydroperoxides, $t$-butylperoxide and hydrogen peroxide, as determined by monitoring the fragments created after exciting the molecule above its dissociation energy.

\section{TETRAMETHYLDIOXETANE}

Tetramethyldioxetane (TMD) is a four membered cyclic peroxide which decomposes over a barrier of $\sim 27 \mathrm{kcal} \mathrm{mol}^{-1}$ to yield, in part, luminescent products. Solution studies find the emission to be primarily from triplet acetone $(30 \%)$ with a smaller quantum yield $(0.5 \%)$ for singlet acetone. ${ }^{7}$ Our low pressure gas phase studies reveal the presence of another, unidentified emitter as well. ${ }^{1 \mathrm{~b}}$ Figure 1 shows the energy level diagram for TMD which is appropriate to our overtone vibration excitation experiment. As described previously, ${ }^{1 \mathrm{a}}$ a $6 \mathrm{~ns}$ pulse from a Nd:YAG laser excited dye laser is shifted by stimulated Raman scattering to the appropriate wavelength regions for exciting $v=3,4$, or 5 of the $\mathrm{CH}$ stretching vibration in TMD. The product luminescence around $460 \mathrm{~nm}$ signals the unimolecular decomposition of a TMD molecule with an excited overtone vibration to form an electronically excited product. Varying the laser 


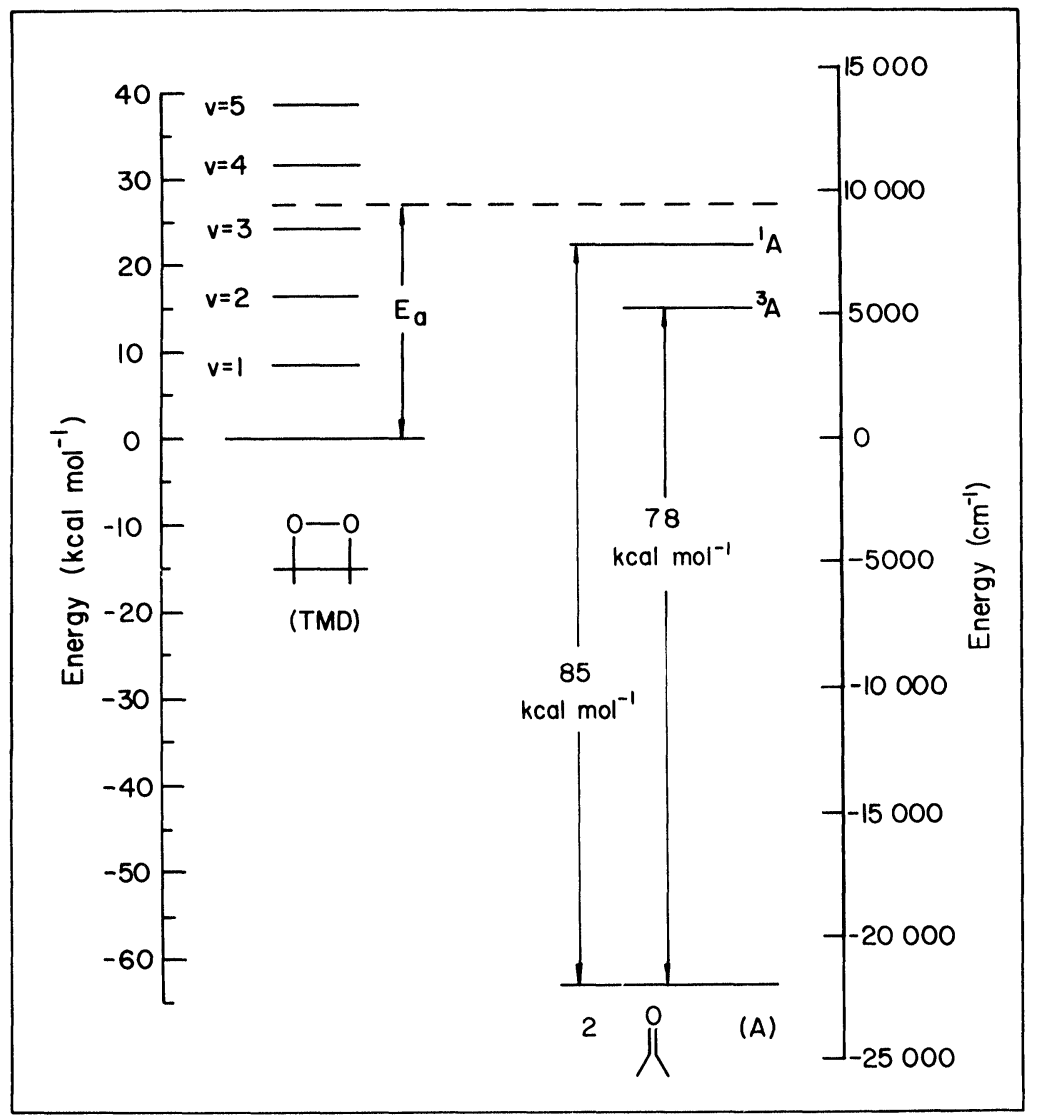

FIGURE 1 Energy level diagram for tetramethyldioxetane.

wavelength maps out an excitation spectrum which reflects the overtone absorption, provided the reaction probability does not vary strongly across the absorption band. Figure 2 shows such spectra for $v=3,4$, and 5 of the $\mathrm{CH}$ overtone vibration along with the conventional infrared absorption spectrum in the $v=1$ region. Photoacoustic experiments by West et $a l^{8}$ confirm that the excitation spectrum accurately mimics the absorption spectrum at least for $v=5$. A photoacoustic measurement and a luminescence excitation 


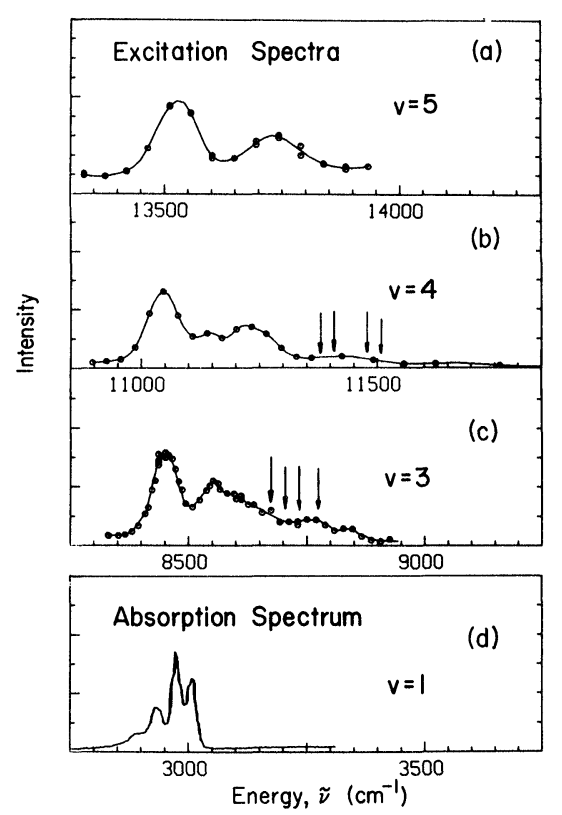

FIGURE 2 Overtone vibration excitation spectra for tetramethyldioxetane (a)-(c) and absorption spectrum of fundamental (d).

experiment yield essentially the same spectrum. (They observe luminescence from the decomposition of cold molecules in a supersonic expansion, as well.)

The overtone vibration spectra all show two maxima with the amount of additional structure being greater for lower vibrational levels. Although all of the hydrogens in TMD are nominally equivalent, local environments which are distinct on the timescale of the excitation process produce the separate maxima in the overtone excitation spectra. An example of this behavior is the overtone absorption spectrum of hexymethylbenzene ${ }^{9}$ in which the methyl hydrogens have two maxima with one arising from hydrogens in the plane of the aromatic ring and the other from those either above or below the plane. These hydrogens experience different electron densities and, consequently, have different bond strengths and characteristic frequencies for the $\mathrm{CH}$ overtone vibration. These local differences are not averaged out by methyl group rotations which are slow 


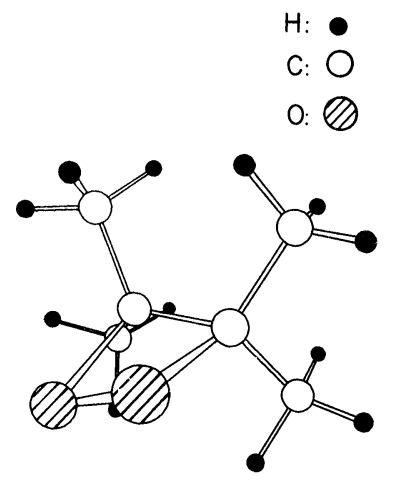

FIGURE 3 Structure of tetramethyldioxetane.

compared to the excitation time. The structure shown in Figure 3 illustrates the situation which can occur in TMD. The structure of the non-planar ring comes from $\mathrm{x}$-ray crystallographic data on a larger dioxetane ${ }^{10}$ and the $\mathrm{CH}_{3}$ distances and geometries are standard parameters. ${ }^{11}$ The methyl hydrogens can either be close to the non-bonded electrons of the oxygen atoms, as illustrated by eight of the hydrogens, or well separated from the oxygens, as the four remaining hydrogens show. The rapid overtone excitation samples the distribution among the possible positions and reflects the preference for certain orientations.

The two maxima in the overtone excitation spectra obey the BirgeSponer relationship individually with $A=3038 \mathrm{~cm}^{-1}$ and $3066 \mathrm{~cm}^{-1}$ and $B=-53 \mathrm{~cm}^{-1}$ and $-55 \mathrm{~cm}^{-1}$ for the low and high energy maxima, respectively, (Table I). The peaks are distinct even at $v=3$, but the more complicated spectra at the lower levels probably reflect the greater normal mode character of the $\mathrm{CH}$ transitions for low overtone vibrations. The arrows in Figure 2 mark the locations of combinations between local modes. The lack of a clear correlation shows that the additional structure is not simply the result of local mode combinations.

Transitions arising from a combination between a vibration of local mode character and one of normal mode character can occur, as well. One of the strongest should be a $\mathrm{CH}$ stretching local mode with a $\mathrm{CCH}$ bending motion since the kinetic energy coupling (as revealed 
TABLE I

Birge-Sponer parameters, $\tilde{\nu}=A v+B v^{2}$

\begin{tabular}{lcc}
\hline \multicolumn{1}{c}{ Molecule } & $A\left(\mathrm{~cm}^{-1}\right)$ & $B\left(\mathrm{~cm}^{-1}\right)$ \\
\hline $\begin{array}{l}\text { Tetramethyldioxetane } \\
\text { (High energy peak) }\end{array}$ & 3066 & -55 \\
$\quad$ Low energy peak) & 3038 & -53 \\
$t$-Butylalcohol & & \\
CH stretch & 2975 & -54 \\
OH stretch & 3725 & -86 \\
$t$-Butylhydroperoxide & & \\
CH stretch & 2975 & -54 \\
OH stretch & 3700 & -94 \\
Hydrogen peroxide & 3701 & -90.5 \\
\hline
\end{tabular}

in the $G$-matrix element) is substantial. ${ }^{12}$ A typical bending frequency is 1200 to $1300 \mathrm{~cm}^{-1}$, and, indeed, we observe transitions at $1300 \mathrm{~cm}^{-1}$ higher energy than the $v=3$ and $v=4$ pure local mode transitions which we assign to the combination of a $\mathrm{CH}$ stretching motion with a $\mathrm{CCH}$ bend. Figure 4 shows the excitation spectrum in the combination region above $v=4$. The multiple peak structure clearly survives in the combination band.

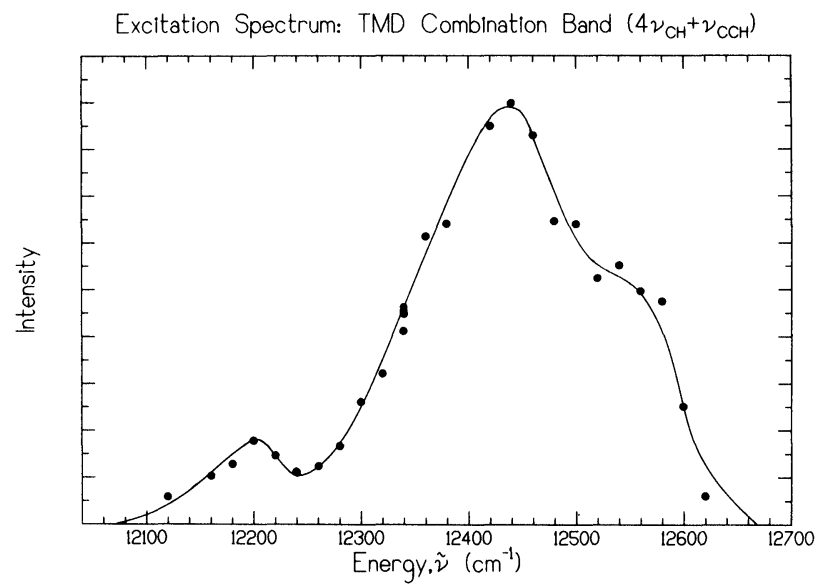

FIGURE 4 Combination band excitation spectrum for tetramethyldioxetane. 


\section{HYDROPEROXIDES}

Hydrogen peroxide and the alkylhydroperoxides are interesting molecules for state-resolved unimolecular decay studies because they produce a ground state $\mathrm{OH}$ fragment which is detectable by laser induced fluorescence and, in the case of the alklhydroperoxides, contain both $\mathrm{CH}$ and $\mathrm{OH}$ bonds in which local mode excitation is possible. The energy level diagram in Figure 5 illustrates the experiment for the case of $t$-butylhydroperoxide. Following laser excitation of the $\mathrm{OH}$ overtone vibration in the parent molecule, a second tunable ultraviolet laser probes individual rotation-vibration states of the $\mathrm{OH}$ fragment by laser induced fluorescence. Varying the time between the two laser pulses maps out the temporal evolution of the fragments for direct determination of the unimolecular decay rate, and varying the probe laser wavelength interrogates individual quantum level populations for analysis of product state distributions. In analogy with the luminescence detection experiments, scanning the excitation wavelength for a fixed delay between the excitation and probe laser

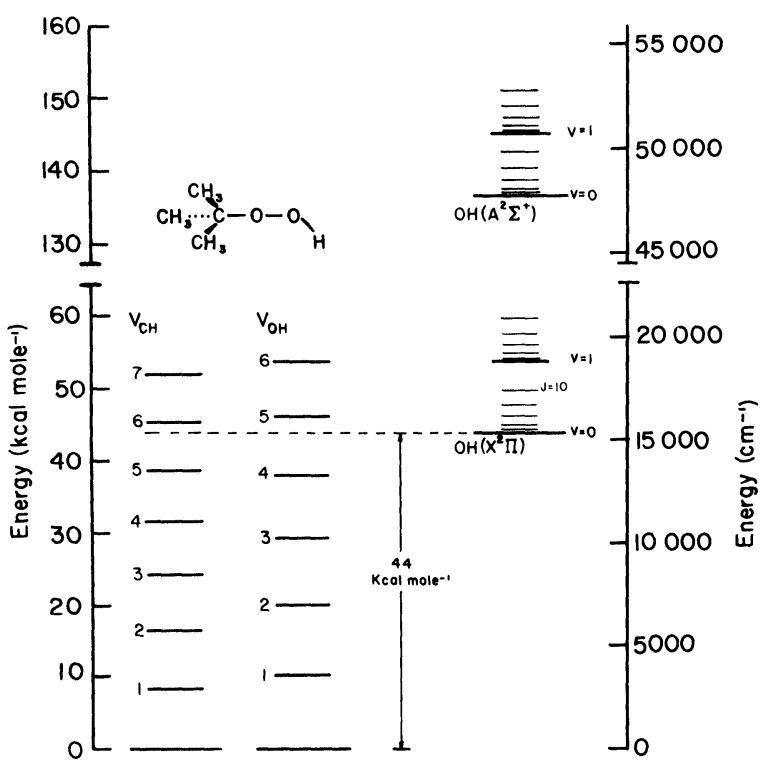

FIGURE 5 Energy level diagram for $t$-butylhydroperoxide. 
pulses and with the probe laser interrogating a particular state of the $\mathrm{OH}$ fragment yields the overtone vibration excitation spectrum for those molecules which decompose to products in the probed state.

The $\mathrm{O}-\mathrm{O}$ bond strength in $t$-butylhydroperoxide $(t-\mathrm{BuOOH})$ is $44 \mathrm{kcal} \mathrm{mol}^{-113}$ while that in hydrogen peroxide $(\mathrm{HOOH})$ is 49.6 $\mathrm{kcal} \mathrm{mol}{ }^{-1},{ }^{14}$ but otherwise the experiment with the latter molecule is quite similar to that illustrated in Figure 5 for $t-\mathrm{BuOOH}$. Of course, there is no $\mathrm{CH}$ stretching vibration available in $\mathrm{HOOH}$, and the decomposition data presented here are for excitation of the $\mathrm{OH}$ stretching overtone vibration in both cases. (The $\mathrm{CH}$ overtone vibration in $t-\mathrm{BuOOH}$ is considerably weaker than the $\mathrm{OH}$ overtone but probably within the sensitivity of our experimental apparatus.)

\section{Tert-butylhydroperoxide}

Tert-butylhydroperoxide is a relatively stable molecule, and we are not limited to studying its overtone vibrations by detecting unimolecular decay fragments. Photoacoustic spectroscopy is an efficient means of detecting overtone absorptions if the molecule of interest has sufficient vapor pressure and does not decompose in the photoacoustic cell. The former is a problem for hydrogen peroxide but not for $t$-butylhydroperoxide, and we have observed both $\mathrm{CH}$ and $\mathrm{OH}$ overtone vibrations below $v=5$ photoacoustically in $t$ - $\mathrm{BuOOH}$. The photoacoustic cell is glass with Brewster angle windows mounted on $\frac{1}{2} \mathrm{~m}$ long side arms and contains a Knowles microphone (BT-1759) with a FET amplifier. A boxcar averager detects the transient electrical signal generated by the microphone in response to the acoustical pulse produced in the sample when the laser is tuned to an overtone vibration.

Figure 6 is the Birge-Sponer plot for the photoacoustically determined pure $\mathrm{CH}$ stretching overtone vibrations in $t$ - $\mathrm{BuOOH}$ (triangles) and in the corresponding alcohol, $t$ - $\mathrm{BuOH}$, (circles). The local $\mathrm{CH}$ oscillators have quite similar Birge-Sponer parameters which are also similar to those for the $\mathrm{CH}$ oscillators in TMD. Table I collects the parameters for all three molecules. These $\mathrm{CH}$ overtone transitions apparently sample similar local environments, as one expects since each of the $\mathrm{CH}$ oscillators is bound to a carbon atom. This comparison is particularly clear for the hydroperoxide and alcohol where the alkyl groups are identical. 


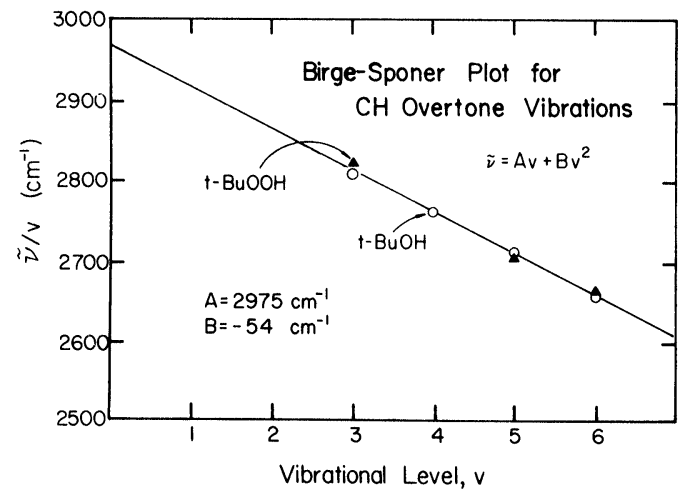

FIGURE 6 Birge-Sponer plot for $\mathrm{CH}$ stretching overtone vibrations in $t$-butylhydroperoxide and $t$-butylalcohol.

The $\mathrm{OH}$ overtone vibration transitions, whose Birge-Sponer plots are shown in Figure 7, exhibit marked differences for $t$-BuOOH and $t$-BuOH. The points are from photoacoustic data except for $v=6$ of $t$ - $\mathrm{BuOOH}$ which is from detection of the unimolecular decomposition fragment. The $\mathrm{OH}$ local mode transitions are higher frequency and slightly less anharmonic in the alcohol than the hydroperoxide. From the Birge-Sponer parameters, we find the nominal dissociation energy

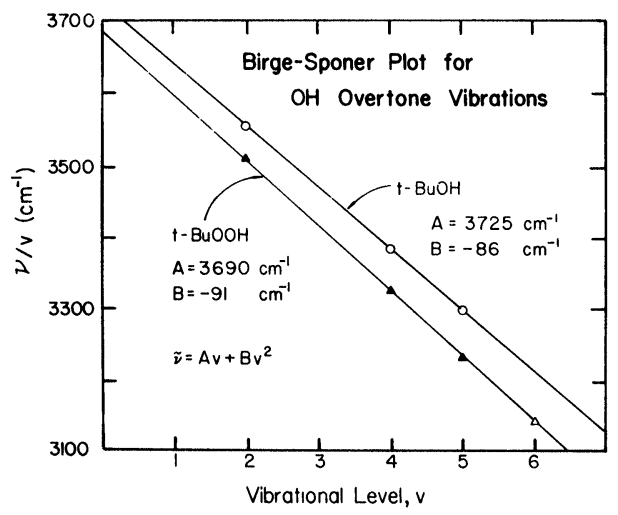

FIGURE 7 Birge-Sponer plot for $\mathrm{OH}$ stretching overtone vibrations in $t$-butylhydroperoxide and $t$-butylalcohol. The open triangle shows the value from decay fragment detection. 
of the $\mathrm{OH}$ bond, $D_{e}=-A^{2} / 4 B$, to be about $10 \%$ higher in the alcohol $\left(115 \mathrm{kcal} \mathrm{mol}^{-1}\right)$ than in the hydroperoxide $\left(104 \mathrm{kcal} \mathrm{mol}^{-1}\right)$. These differences reflect the $\mathrm{OH}$ local environments which, in contrast to the situation with $\mathrm{CH}$, differ strongly between the two molecules. In the alcohol the $\mathrm{OH}$ oscillator is attached to a carbon atom while in the hydroperoxide it is bound to an oxygen atom.

\section{Hydrogen peroxide}

The low vapor pressure of $\mathrm{HOOH}$ in the photoacoustic cell prevents our taking overtone absorption spectra directly, but extrapolation of conventional data on low overtone vibrations $(v=2$ and $v=3)$ provides an estimate of the wavelength region in which the $v=6$ transition of the $\mathrm{OH}$ local mode occurs. Figure 8 is a Birge-Sponer plot for $\mathrm{HOOH}$ where the point at $v=1$ is the average of the frequencies of the normal mode asymmetric and symmetric stretches and those at $v=2$ and $v=3$ are averages of closely spaced rotational origins in the respective overtones. ${ }^{15}$ The solid line, which is fit to the points for $v=2$ and $v=3$, yields the Birge-Sponer parameters shown in Table

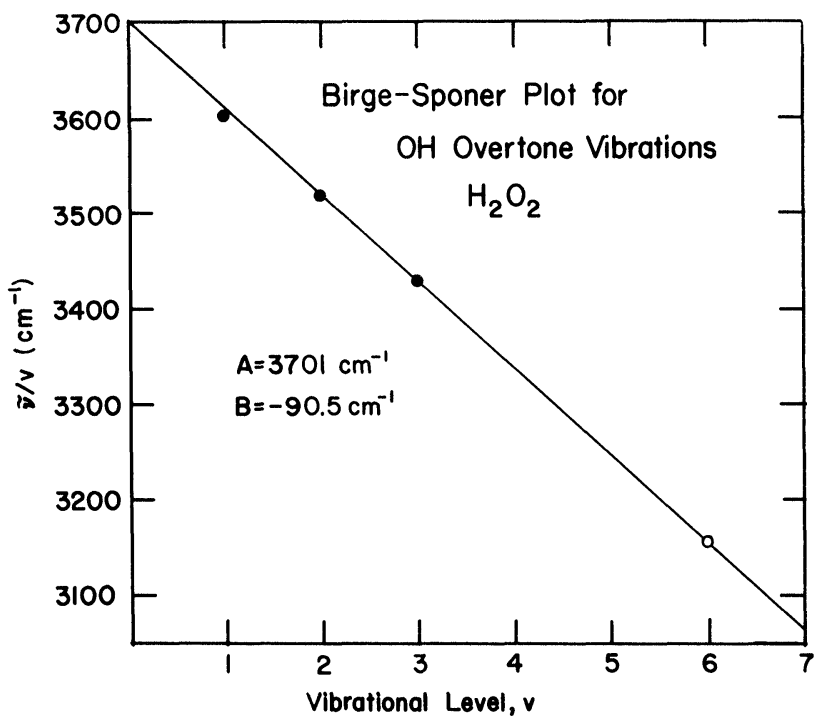

FIGURE 8 Birge-Sponer plot for $\mathrm{OH}$ stretching overtone vibrations in $\mathrm{HOOH}$. The open symbol shows the value from decay fragment detection. 
I and accurately predicts the frequency of the $v=6 \mathrm{OH}$ stretch in $\mathrm{HOOH}$. Our measurement using product detection in the overtone vibration initiated unimolecular decomposition of $\mathrm{HOOH}$ is the open circle.

Scanning the excitation laser through the wavelength region predicted for $v=6$ by Birge-Sponer plot while probing the $Q_{1}(4)$ transition in the $\mathrm{OH}$ fragment yields the spectrum shown in Figure 9a. The largest feature is the $v=6$ local mode transition as predicted by the Birge-Sponer plot. There is sharp, reproducible structure on this transition which probably arises from rotational progressions which are apparent in lower transitions. Clearly the overtone transition is not homogeneously broadened over its entire width. The other small

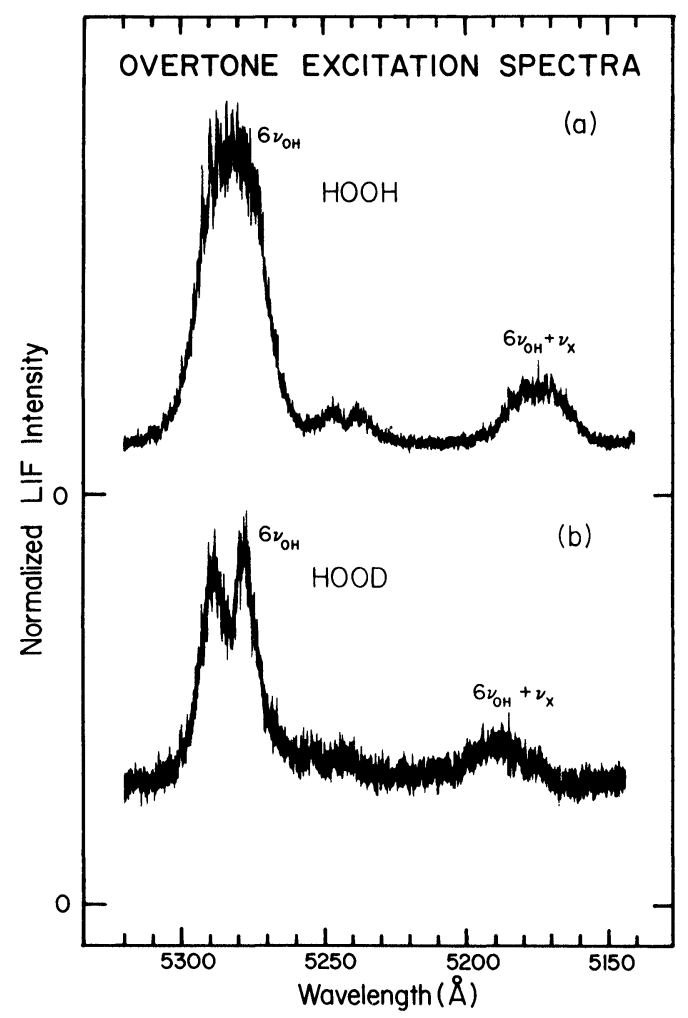

FIGURE 9 Overtone vibration excitation spectra for (a) HOOH and (b) HOOD. 
features are likely to be combinations of local mode and normal mode transitions. The most prominent of these lies about $385 \mathrm{~cm}^{-1}$ above the main transition. A possible normal mode $\left(\nu_{x}\right)$ which is combined with $6 \nu_{\mathrm{OH}}$ in this case is the torsion involving all four atoms. Because of different size barriers to cis and trans rotation about the $\mathrm{O}-\mathrm{O}$ bond, the structure of the torsional levels is complicated, ${ }^{16}$ but one of the strongest torsional transitions occurs at $370 \mathrm{~cm}^{-1} .16 \mathrm{c}$

The spectrum in the same region for partially deuterated hydrogen peroxide, $\mathrm{HOOD}$, appears in Figure $9 \mathrm{~b}$. Adding $\mathrm{D}_{2} \mathrm{O}$ to the $90 \%$ $\mathrm{HOOH} / \mathrm{H}_{2} \mathrm{O}$ mixture and redistilling it to increase the concentration of peroxide produces an equilibrium mixture of $\mathrm{HOOH}, \mathrm{HOOD}$, and DOOD, and we identify the partially deuterated species by exciting in the $\mathrm{OH}$ overtone vibration region and detecting the $\mathrm{OD}$ fragment $\left(Q_{1}(6)\right)$. The substitution of a deuterium dramatically changes the main peak. Its center frequency remains the same, but it splits into two components. The combination features move to lower frequency, supporting their assignment as including motion of the deuterated end of the molecule.

These overtone vibration studies illustrate the utility of overtone vibration excitation for initiating state-resolved unimolecular decay experiments and demonstrate the sensitivity possible using decay fragment detection as a monitor of local mode excitation. In addition to being a fruitful approach to studying unimolecular decay dynamics, this technique is a sensitive means of obtaining local mode spectra.

\section{References}

1. (a) B. D. Cannon and F. F. Crim, J. Chem. Phys. 75, 1752 (1981). (b) B. D. Cannon and F. F. Crim, J. Am. Chem. Soc. 103, 6722 (1981).

2. T. R. Rizzo and F. F. Crim, J. Chem. Phys. 76, 2754 (1982).

3. T. R. Rizzo, C. C. Hayden and F. F. Crim, Faraday Disc. Chem. Soc. (1983), in press.

4. B. R. Henry, Acct. Chem. Res. 10, 207 (1977).

5. M. L. Sage and J. Jortner, Adv. Chem. Phys. 47, 293 (1981).

6. R. L. Swofford, M. E. Long and A. C. Albrecht. J. Chem. Phys. 65, 179 (1976).

7. (a) N. J. Turro, P. Lechtekn, N. E. Schore, G. Schuster, H.-C. Steinmetzer and A. Yekta, Acc. Chem. Res. 7, 97 (1974). (b) W. Adam, Adv. Heterocyclic Chem. 21, 437 (1977).

8. G. A. West, R. P. Mariella, Jr., J. A. Pete, W. B. Hammond and D. F. Heller, J. Chem. Phys. 75, 2006 (1981).

9. B. R. Henry and W. R. A. Greenlay, J. Chem. Phys. 72, 5516 (1980).

10. H. Nurman, J. Wieringa, H. Wynberg, J. Hess and A. Vos, Chem. Soc. Commun. 591 (1977). 
11. Chemical Society Special Publication No. 11. Tables of Interatomic Distances and Configuration in Molecules and Ions. (The Chemical Society, London, 1958).

12. E. L. Sibert, III, W. P. Reinhardt and J. T. Hynes, Chem. Phys. Lett. 92, 455 (1982). 13. J. A. Kerr, Chem. Rev. 66, 465 (1966).

14. H. Okab in Photochemistry of Small Molecules (John Wiley, New York, 1978) p. 282.

15. P. A. Guiguère, J. Chem. Phys. 18, 88 (1949).

16. (a) P. Helminger, W. C. Bowman and F. C. DeLuca, J. Mol. Spectrosc. 85, 120 (1981). (b) L. Zumwalt and P. A. Guiguère, J. Chem. Phys. 9, 458 (1941). (c) R. H. Hunt, R. A. Leacock, C. W. Peters and K. T. Hecht, J. Chem. Phys. 42, 1931 (1965). (d) R. L. Redington, W. B. Olson and P. C. Cross, J. Chem. Phys. 36, 1311 (1962). 